\title{
Hyperandrogenic States in Pregnancy
}

\author{
N. KAŇOVÁ ${ }^{1}$, M. BIČÍKOVÁ ${ }^{1}$ \\ ${ }^{1}$ Institute of Endocrinology, Prague, Czech Republic
}

Received September 6, 2010

Accepted October 8, 2010

On-line November 29, 2010

\begin{abstract}
Summary
Hyperandrogenic states in pregnancy are almost always the result of a condition that arises during pregnancy. The onset of virilization symptoms is often very fast. The mother is protected against hyperandrogenism by a high level of SHBG, by placental aromatase and a high level of progesterone. The fetus is protected from the mother's hyperandrogenism partly by the placental aromatase, that transforms the androgens into estrogens, and partly by SHGB. Nevertheless there is a significant risk of virilization of the female fetus if the mother's hyperandrogenic state is serious. The most frequent cause of hyperandrogenic states during pregnancy are pregnancy luteoma and hyperreactio luteinalis. Hormonal production is evident in a third of all luteomas, which corresponds to virilization in 25-35\% of mothers with luteoma. The female fetus is afflicted with virilization with two thirds of virilized mothers. Hyperreactio luteinalis is created in connection with a high level of hCG, e.g. during multi-fetus pregnancies. This condition most frequently arises in the third trimester, virilization of the mother occurs in a third of cases. Virilization of the fetus has not yet been described. The most serious cause of hyperandrogenism is represented by ovarian tumors, which are fortunately rare.
\end{abstract}

\section{Key words}

Hirsutism • Hyperandrogenemia • Virilism • Luteoma • Cyst

\section{Corresponding author}

N. Kaňová, Institute of Endocrinology, Národní 8, 11694 Prague 1, Czech Republic. E-mail: nkanova@endo.cz

\section{Introduction}

The incidence of hyperandrogenic states during pregnancy is relatively low. Hyperandrogenic states during pregnancy are almost always the result of a condition that arises over the course of the pregnancy, since a high level of androgens that occurs before conception causes anovulation and leads to infertility.

The clinical symptoms of hyperandrogenism during pregnancy are not different from those during nonpregnancy. This most frequently leads to an occurrence of hirsutism in androgen-dependent areas of predilection, such as the upper lip, chin, linea alba, groin, thigh and chest. There is often acne on the face, shoulders, back and chest. With some women there occurs hair loss with ensuing hair thinning in the temporoparietal area. During pregnancy we relatively frequently see the rapid onset of masculine symptoms such as clitoromegaly and deepening voice caused by a larger larynx. Some hyperandrogenic symptoms are fully reversible, such as acne and hair loss. On the contrary, hirsutism, deepening voice and clitoromegaly are only partially reversible. Therapy for these symptoms is possible only after removing the causes of hyperandrogenism. Laser treatment is currently used to treat hirsutism. In addition to phoniatric care, the vocal chords can be operated on to rectify a deep voice.

\section{Physiological changes of androgens during pregnancy}

During pregnancy extensive changes take place in the mother's organism that enables the successful development of the fetus. The high level of estrogens causes an increase in protein synthesis in liver and with it an increase in specific binding proteins such as sex hormone-binding globulins (SHGB), whose level rises from the beginning of the pregnancy. 


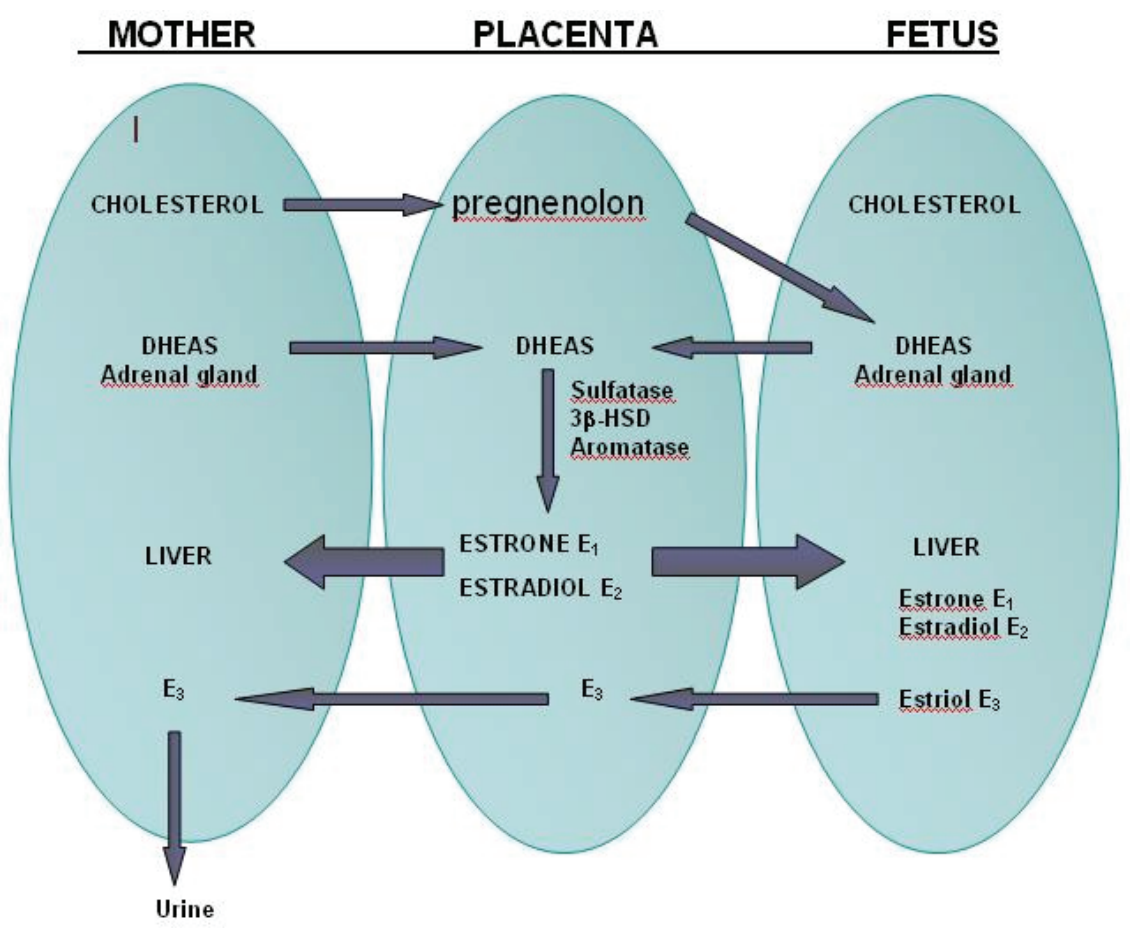

Fig. 1. Estrogens in the placenta.

During pregnancy the synthesis of dehydroepiandrosterone (DHEA) in the adrenal glands increases significantly. Its sulphate (DHEAS) is the main precursor of the synthesis of steroid hormones in the placenta, which is why the plasmatic level of both of these androgens drops by nearly half during pregnancy (Milewich et al. 1978). Due to the increased level of SHBG from the start of pregnancy there occurs a change in the ratio of the free and bound testosterone. The level of the free testosterone is due to the higher binding to SHBG up to the 28th week of pregnancy lower than during non-pregnancy; then, however, it rises and peaks in the third trimester (Rivarola et al. 1968, Milewich et al. 1978, Barbieri 1999). Feedback set for levels of free testosterone leads to increased production of testosterone so that the total testosterone at the end of the first trimester reaches levels common with men.

\section{Protection of the mother from excess of androgens during pregnancy}

An excess of androgens produces lower clinical manifestations during pregnancy than with non-pregnant women. The main reason is the high level of SHBG which is able to bind most sex hormones. Affinity is greatest with dihydrotestosterone (DHT) that is bound from $59 \%$. This is followed by testosterone (44\%), estradiol $(19.6 \%)$ and estrone $(7.4 \%)$. The remaining steroids have a very weak and therefore insignificant binding; less than five percent from the total amount is bound to SHBG (DHEA, androstenedione and estriol). Due to the high binding of strong androgens during pregnancy the level of free, biologically effective androgens during hyperandrogenism remains without change for a long time.

Another factor is the aromatase cytochrome P 450 present in the placenta. The placenta is fully equipped to deal with enzymes, allowing for the synthesis of steroid hormones. In the human placenta there is not expressed the steroid 17 $\alpha$-hydroxylase (CYP17), therefore $C_{21}$ steroids cannot be converted to $C_{19}$ steroids in trophoblast. Besides the above-mentioned aromatase a basic role is played by the placental steroid sulfatase,

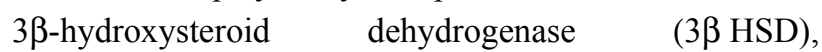
17 $\beta$-hydroxysteroid dehydrogenase (17 $\beta$ HSD) and others. As a precursor of the synthesis of steroid hormones in the placenta, there is used dehydroepiandrosterone sulfate (DHEAS), whose source is partly the fetal adrenal glands and to a lesser degree the mother's adrenal glands. DHEAS is desulfurylated by steroid sulfatase. Free DHEA is converted by $3 \beta$ HSD and $17 \beta \mathrm{HSD}$ to androstenedione and testosterone, respectively. Androstenedione is converted by placental aromatase to estrone which is converted by $17 \beta$ HSD to estradiol. These estrogens pass over the placental barrier to the mother's circulation and the fetus where liver uptake occurs. Enzymatic apparatus in fetal liver create estriol, which is the main pregnancy estrogen that is, after 
crossing over the placental barrier to the mother's circulation, it is filtered through the kidneys and gets into urine in high levels (Fig. 1). Thanks to this it can be used as an indicator of the function of the fetal-placental unit in pregnancy. During a successful pregnancy its level rises smoothly up to the time of birth. Under normal circumstances aromatization of the androgens require only roughly $1 \%$ functional capacity of placental aromatase. If the mother's organism or the fetus is flooded with androgens, the functional capacity of this enzyme increases in significance.

Last but not least, a high level of progesterone contributes to the reduction of the clinical manifestation of hyperandrogenism during pregnancy. This competes with androgens for binding places to androgen receptors (Clement 1993, Choi et al. 2000).

\section{Protection of the fetus from the mother's hyperandrogenism}

The most important factor protecting the fetus from a mother's hyperandrogenism is the placental aromatase's cytochrome P 450 (Illingworth et al. 1992), whose mechanism of effect was described above. However, this enzyme has a certain limitation. For an extreme amount of androgens, e.g. for ovary tumors, the functional capacity of aromatase is exceeded and the androgens are not sufficiently removed from the circulation (Shortle et al. 1987). What's more, placental aromatase is not capable of aromatising dihydrotestosterone (DHT), whose level often rises in hyperandrogenic states, particularly with ovarian etiology. Increased levels lead to relatively easy virilization of the female fetus (Norwitz 2004).

SHBG also represents substantial protection of the fetus from the mother's hyperandrogenism.

\section{Risk of virilization of the female fetus}

Virilization of the female fetus occurs most frequently from fetal causes (congenital adrenal hyperplasia, $\mathrm{CAH}$ ). Other causes are less frequent. The risk of virilization of the female fetus during the mother's hyperandrogenism depends on the period of the beginning of the hyperandrogenism - the severest affliction of the female fetus's genitalia occurs during hyperandrogenism in the first trimester. Moderate mother's hyperandrogenism in the second and third trimester does not necessarily result in virilization of the fetus; on the contrary severe hyperandrogenism causes malformation of the genitalia in this period as well. Other factors (such as functionality of the placental aromatase), of which some are unknown, also influence whether virilization of the female fetus occurs.

\section{Etiology of hyperandrogenic states in pregnancy}

\author{
1/ Ovarian - tumor \\ - non-tumor \\ 2/ Adrenal - tumor \\ - non-tumor \\ 3/ Fetal - Fetal Aromatase Deficiency (FAD) \\ 4/ Iatrogenic
}

The etiology of hyperandrogenic states can be divided into the four aforementioned groups. The most frequent cause is a non-tumor ovarian condition (pregnancy luteoma, hyperreactio luteinalis, PCOS). In contrast, adrenal tumor causes are extremely rare (Dahl et al. 2008). A mother's hyperandrogenism caused by fetal etiology is created with a deficiency of placental aromatase. Iatrogenic hyperandrogenism in a pregnant woman occurs with the use of preparations containing androgens or some progestins (e.g. danazol).

\section{Ovarian tumor causes}

The cause of hyperandrogenism with a mother can be tumors from gonadal mesenchyme, such as granulosa/theca cell tumors, thecoma and tumors from Sertoli-Leydig cell (Young et al. 1984). Tumors from functional tissue are relatively rarely the cause of hyperandrogenism of the pregnant. Those described include, e.g., cystadenocarcinoma, Brenner tumor and cystadenoma (Pascal and Grecco 1988). Krukenberg tumor can also be manifested via hyperandrogenism; this is the most frequent bilateral "potatolike" metastatic affliction of ovaries by a muciparous cancer, coming most frequently from the digestive tract or the mamma). Virilization occurs with $80-100 \%$ of those afflicted. If the cause of hyperandrogenism is an ovarian tumor, the hyperandrogenism state will continue even after the birth, in contrast to benign ovarian conditions (e.g. pregnancy luteoma, hyperreactio luteinalis) the spontaneously diminish following the birth. The incidence of ovarian tumors during pregnancy is described by various authors in a range of $1: 112$ to $1: 2200$ (Vulink et al. 2004). The 
most frequent causes are dermoid cysts that make up $48 \%$ of cases and are usually hormonally inactive (Ueda and Ueki 1996). The rate of incidence of malignant tumors is $2.4 \%-7.9 \%$ (Manganiello et al. 1995). The share of hormonally active tumors has not been systematically researched.

\section{Ovarian non-tumor causes}

Hyperandrogenic states during pregnancy are most frequently caused by any of three benign ovarian conditions. The first two most frequent are pregnancy luteoma and hyperreactio luteinalis (McClamrock and Adashi 1992). The clinical manifestation usually occurs in the second trimester; after the birth it spontaneously disappears, but it often reappears during later pregnancies (Joshi and Dunaif 1995). The most frequent cause of hyperandrogenism in the first trimester is PCOS (Vulink et al. 2004). Virilization is often only slight (Ben-Chetrit and Greenblatt 1995), it spontaneously diminishes 3-4 months after the birth.

\section{Pregnancy luteoma}

Pregnancy luteoma was first described in 1963 (Sternberg 1963). This is a non-tumor lesion of the ovaries that resembles in its appearance ovarian tumors (Wang et al. 2005). It is described as a large solid nontumor mass in the area of the ovary, most frequently around $6-10 \mathrm{~cm}$, but luteomas have been described from microscopic to $20 \mathrm{~cm}$ large (Clement 1993). It typically has a multi-nodular appearance of yellow or brownishyellow colour, often with dark red hemorrhagic deposits (Kao et al. 2005). To date there has been just over 200 cases described in writing, but the occurrence is most likely more frequent since there also exists the so-called sub-clinical luteomas that are completely asymptomatic.

Luteomas are created microscopically by groups of luteinised cells with large eosinophilic cytoplasm and regular nucleuses (Spitzer et al. 2007). From a pathogenetic standpoint this consists of a solid proliferation of luteinised cells of tissue under the influence of the normal level of human chorionic gonadotropin (hCG) (Tan et al. 2008).

Pregnancy luteomas are always benign. They can be numerous, in 30 to 50 percent of cases they are bilateral (McClamrock and Adashi 1992, Clement 1993). They mostly occur in women in their third to fourth decade of life, more frequently with multiparas
(Azmoun and DiSalvo 1999). The greatest frequency was recorded with the Negro race (Joshi and Dunaif 1995), followed by women with pre-existing PCOS. Benign luteoma must not be confused with ovarian tumors. With the secretion of a small amount of androgens that do not cause manifest hyperandrogenism, pregnancy luteoma can by entirely asymptomatic - i.e. subclinical luteoma. It is sometimes found during an ultrasound or with Caesarean section births.

Hormonal production is described for $30 \%$ of luteomas of pregnancy. Androgens are created for $25 \%$ of luteomas (Spitzer et al. 2007), of which 10-50\% are clinically manifest. If manifest virilization of the mother occurs the female fetus has a 60-70\% likelihood that it will be virilized too (Spitzer et al. 2007). The remaining $30-40 \%$ of unborn girls are protected thanks to placental aromatase. In a lesser degree estrogens and progesterones are synthetized in luteomas. Clinical signs of virilization of the mother are described in $25-35 \%$ of cases (Tan et al. 2008). With 2/3 of virilized mothers there occurs virilization of the female fetus (Spitzer et al. 2007).

For women with pregnancy luteoma there occurs a delay in the onset of lactation following the birth - with most the length is one week (Dahl et al. 2008). Under normal circumstances lactation starts 3040 hours after the birth (Betzold et al. 2004). Hyperandrogenism has a suppressive effect on the mammary gland. This was therapeutically used in the past to stop lactation for women following abortions, etc., when a combination of high doses of estrogen was used in combination with androgens (Kochenour 1980). This therapeutic process was abandoned due to the high risk of thromboembolism. For lactation to start the level of testosterone must drop below $10.4 \mathrm{nmol} / 1$ (Betzold et al. 2004). The stimulation of the mammary gland must also be maintained by frequent breast feeding and the breast pump.

Pregnancy luteoma can be the cause of acute abdomen caused by ovarian torsion, tumor rupture or bleeding (Tan et al. 2008). Luteomas causing suppression of surrounding organs, such as compression of the ureters with ensuing obstructive uropathy have been described (Wang et al. 2005).

Normal imaging methods are used for morphological depiction. Ultrasonography of the lesser pelvis is during pregnancy of a lower quality due to the enlarging uterus, which is why an magnetic resonance 
(MRI) is preferred (Kao et al. 2005). During ultrasound or MRI examination pregnancy luteoma is $90 \%$ shown as a solid formation in the lesser pelvis. In the case of necrotical changes, it can have a cystic character and then is difficult to tell apart from a tumor.

Pregnancy luteoma spontaneously regresses over the course of the first three months (Choi et al. 2000, Wang et al. 2005). The level of androgens drops during the first two weeks following the birth (Clement 1993). Depending on the level of virilization of the mother the modification of clinical symptoms lasts two to six months, although some symptoms are not fully reversible (clitoromegaly, deep voice). Although pregnancy luteoma disappears after the birth, its reccurrence is to be expected during later pregnancies (Holt et al. 2005). There are cases described in which there occurred virilization of the genitalia of female fetuses with the reccurrence of luteomas during repeated pregnancies accompanied by hyperandrogenism (Wang et al. 2005, Spitzer et al. 2007). A possible solution that is offered is intrauterine insemination with pre-selection of Y-spermatozoa for women with a medical history of manifest pregnancy luteoma with virilization of the female fetus (Chen et al. 2009).

Pregnancy luteoma is a "self-limited disease", which is why surgery is not usually indicated. In the case in which an asymptomatic formation is randomly discovered in the vicinity of the ovaries in the third trimester or during the birth, there is not surgical treatment on the spot and only observation is indicated.

Acute abdomen is a clear indication of surgical removal of the luteoma. A relative indication for surgery can be the discovery of a tumor-like mass in the area of the lesser pelvis in the 2 trimester with a virilized woman, or a solid unilateral enlargement of the ovary during pregnancy; the risk of malignancy is in these cases $50 \%$ (Quagliarello and Blaustein 1985, McClamrock and Adashi 1992). Surgery should also be considered if a cystic formation larger than $5 \mathrm{~cm}$ is discovered that does not reduce in size during the pregnancy.

If a surgical removal of the luteoma is necessary, the operation should be timed for the second trimester. A high occurrence of miscarriages is described with operations in the first trimester; in the third trimester it often provokes a premature birth. The method of selection is an explorative laparotomia with salpingooophorectomy. The operation is done to remove the formation, protect the developing fetus and preserve the fertility of the woman in the future.

\section{Hyperreactio luteinalis}

Hyperreactio luteinalis is the cystic enlargement of the ovaries caused by numerous theca-luteina cysts. It arises in connection with a high level of the choriostimulating hormone (hCG) over $300000 \mathrm{IU}$, which occurs with a multiple-fetus pregnancy, molar pregnancy or choriocarcinoma (Kurman 1987, Shortle et al. 1987). It is assumed that a certain pathological state of ovaries (e.g. PCOS or multiple corpora lutea) is the cause of internal sensitivity of the ovaries to gonadotropic effects (Check et al. 2000), that has as its consequence the hypertrophy and luteinization of the theca interna cells. With this the woman becomes more susceptible to the ovarian hyperstimulation syndrome (OHSS) (Schenker and Ezra 1994). Considerable luteinization and hypertrophy of theca interna cells is histologically demonstrated in ovaries. This disease is considered to be a form of hyperstimulation of the ovaries. In $70 \%$ of cases it arises in the third trimester, or even soon after the birth (Wajda et al. 1989) most frequently in connection with molar pregnancy, or even with choriocarcinoma. In $16 \%$ it occurs in the first trimester (Foulk et al. 1997), then it is very difficult to differentiate it from spontaneous ovarian hyperstimulation syndrome arising without therapeutic use of follicle-stimulating medicaments (Zalel et al. 1992, Ayhan et al. 1996). Hyperreactio luteinalis is usually found with first-time mothers, more with women of the white race. It is often a bilateral occurrence. It is most frequently asymptomatic. Sometimes it clinically imitates ovarian hyperstimulation syndrome with increased permeability of membranes which manifests itself through swelling, ascites or hydrothorax (Foulk et al. 1997). Anasarca has also rarely been described (Lambers and Rosenn 1996). Patients often described subjective complaints such as abdominal discomfort, breathlessness, abdominal pain (Al-Ramahi and Leader 1999). In $30 \%$ of the cases there occurs hyperandrogenism in the mother. Virilization of the fetus has not yet been described (Norwitz 2004).

\section{Fetal cause - placental aromatase deficiency (FAD)}

Placental aromatase deficiency arises in genetically conditioned (autosomal recessive inheritance) mutation of the placental CYP 19 gene (Harada et al. 1992). Other enzymes remain functional in the placenta, which is why the weak androgen DHEAS is further subject to conversion to stronger androgens such as 
testosterone and androstenedione, but a final aromatization to estrogens is missing. With this there occurs an accumulation of all androgens, which is made clinically manifest as virilization of the mother in the second half of the pregnancy.

In a lab examination we find with the mother extremely low estradiol and estriol and a significantly higher level of testosterone. With a female fetus there occurs severe virilization of the genitalia (pseudohermaphroditism femininus); infants of the male sex are born with normal genitalia. To verify the diagnosis a determination of the activity of aromatase in the frozen placental tissue is made.

\section{Adrenal causes}

With some tumors of the adrenal glands producing androgens (carcinoma of the adrenal cortex, adenoma of the adrenal cortex) there can occur hyperandrogenism of the pregnant woman. This, however, is an extremely rare condition during pregnancy. Hyperandrogenism can also accompany Cushing syndrome. Forms, related to pregnancy appear at the start of pregnancy and spontaneously subside after the birth - with some patients even repeatedly (Wy et al. 2002). The cause can be irregular secretion of corticotropin releasing hormone $(\mathrm{CRH})$ in the placenta or the presence of "afunctional" adenomas in the cortex of the adrenal glands that contain receptors with aberrant activity for hCG, vasopressin, etc.

\section{Clinical examination of a patient with hyperandrogenism during pregnancy}

It is necessary to anamnestically assess the period of the onset of symptoms (first - third trimester), the speed of their progress, the regularity and character of the menstrual cycle before pregnancy, the use of progestins, androgens and other medicaments during pregnancy.

During the physical examination we assess BMI, blood pressure, we quantify the level of hirsutism, the presence of signs of virilization, cushingoid traits and we supplement this with a vaginal and rectal examination. Laboratory examination is focussed on determining the level of testosterone, androstenedione, DHEA and DHEAS, dihydrotestosterone, SHBG, cortisol and estrogens (estradiol, estriol).

Of the various imaging methods the method of choice is the ultrasound examination of the adrenal glands and ovaries that enables an assessment of the size of the formation, the distinguishing of the solid and cystic character, unilateral and bilateral affliction, or possible changes in the sex of the fetus as the case may be. During pregnancy, however, it might not be sufficiently precise, which is why a magnetic resonance can be used to assess the local growth of the tumor, or the presence of metastasis.

\section{Short case report}

For illustrative purposes we are presenting a short case study of a 31-year-old patient on whom a thick growth of hair appeared on the neck and chin and maletype hair grew on the abdomen. Roughly a month before birth there occurred a deepening of her voice, acne appeared on her face and back. Otherwise the course of the pregnancy was physiological; spontaneous birth took place in a regular timeframe, a full-term boy weighing $3140 \mathrm{~g}$ and $49 \mathrm{~cm}$ in size with an APGAR score of 8-10-0 was born. During the pregnancy the patient gained a total of $7 \mathrm{~kg}$, of which $6 \mathrm{~kg}$ she immediately lost after the birth. The onset of lactation did not occur.

The Table 1 shows the development of hormonal levels in the first three weeks following the birth. The high level of testosterone and androstenedione were normalized by the $21 \mathrm{st}$ day after the birth. The other biochemical examination did not turn up any concerns, no tumor markers. A sonographic examination was unable to show the adrenal glands after the birth, the CT of the adrenal glands 21 days after the birth was normal. A sonographic examination on the seventh day after the birth showed an enlarged right ovary $(51 \times 36 \times 17)$ of an atypical appearance, while the left ovary was not enlarged. Normalization of the sonographic discovery on the right ovary occurred two months after the birth.

The acne diminished a month after the birth. The increased hair growth lasted another three months, four months after the birth there occurred a massive outbreak of hair on the face, the patient plucked the remaining few hairs from her chin. Hirsutism on the abdomen and on the groin continued in a moderate form. The deep voice apparently improved, but continued to be deeper than before the pregnancy (Fig. 2). 
Table 1. Changes of hormonal levels during the three weeks after birth.

\begin{tabular}{|c|c|c|c|}
\hline & day 4th & day 13th & day 21st \\
\hline Testosterone & 26 & 6.73 & 1.59 \\
\hline $\mathrm{nmol} / \mathrm{l}$ & (norm $0.24-3.12$ ) & (norm $0.40-3.00$ ) & (norm $0.40-3.00)$ \\
\hline Androstenedione & 8.26 & 6.53 & 4.57 \\
\hline $\mathrm{nmol} / \mathrm{l}$ & (norm 1.42-5.34) & (norm 1.50-5.40) & (norm.50-5.40) \\
\hline$D H E A-S$ & 3.5 & 3.69 & $\mathbf{3 . 7 0}$ \\
\hline umol/l & (norm 0.269-8.98) & (norm 1.80-9.70) & (norm 1.80-9.70) \\
\hline$S H B G$ & 306 & 157 & 85.4 \\
\hline $\mathrm{nmol} / \mathrm{l}$ & (norm 20-118) & (norm 43.2-94.8) & (norm 43.2-94.8) \\
\hline cortisol & 823 & 555 & 677 \\
\hline nmol/l & (norm 260-720) & (norm 135-607) & (norm 135-607) \\
\hline estradiol & 4.6 & 0.128 & 0.068 \\
\hline nmol/l & (norm 0.183-0.917) & (norm 0.110-1.290) & (norm $0.110-1.290$ ) \\
\hline prolactin & 78.6 & 17.5 & 17.2 \\
\hline$n g / m l$ & (norm 2.8-20) & (norm 4.8-23.4) & (norm 4.8-23.4) \\
\hline$L H$ & undetectable low & 0.4 & 0.2 \\
\hline$I U / l$ & & (norm 2.0-90.0) & (norm 2.0-90.0) \\
\hline FSH & 0.2 & 0.1 & 1.8 \\
\hline$I U / l$ & & (norm 2.0-30.0) & (norm 2.0-30.0) \\
\hline progesterone & 19 & 2.02 & 2.84 \\
\hline $\mathrm{nmol} / \mathrm{l}$ & (norm $7.9-79.3$ ) & (norm $0.30-80.00$ ) & (norm $0.30-80.00)$ \\
\hline $17 \alpha$ hydroxyprogesterone & & 2.90 & 2.51 \\
\hline nmol/l & & (norm $0.30-4.50)$ & (norm $0.30-4.50)$ \\
\hline dihydrotestosterone & & 1.11 & 0.73 \\
\hline nmol/l & & (norm 0.10-0.90) & (norm $0.10-0.90)$ \\
\hline
\end{tabular}

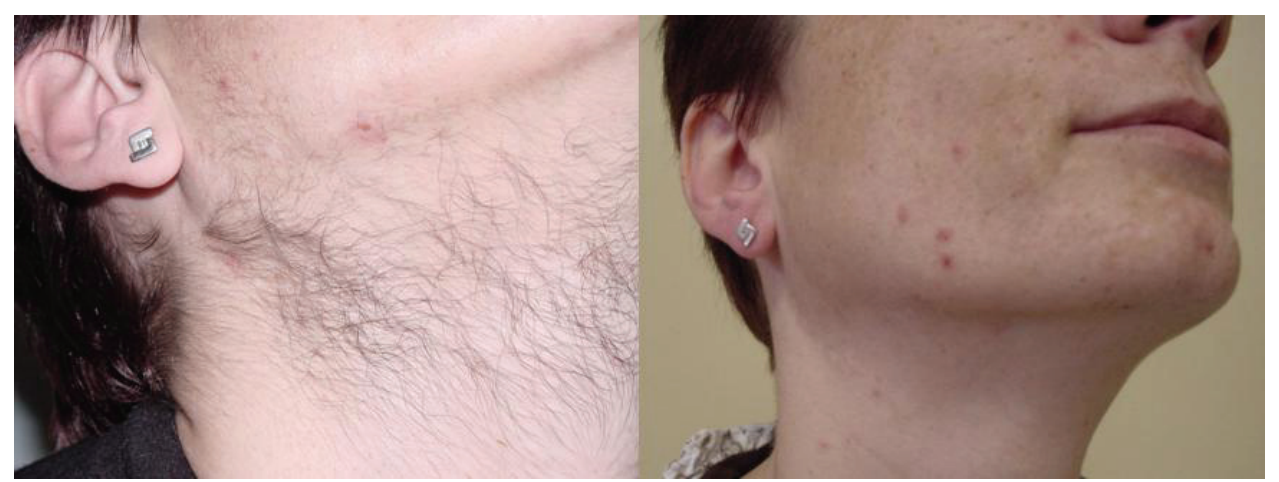

Fig. 2. Hirsutism on the neck just before birth and 4 months after birth.

According to the clinical course, the lab examinations and imaging methods we consider the most likely cause of hyperandrogenism during the patient's pregnancy to be pregnancy luteoma. Although there occurred spontaneous rectification of all finds, there remains the risk of a reccurrence of hyperandrogenism in a later pregnancy and the possible risk of virilization of the female fetus that could lead to lifelong stigmatisation of the hitherto unborn girl.

\section{Clinical examination and conclusion}

For a pregnant woman with signs of a hyperandrogenic state it is necessary to anamnestically assess the period of the onset of symptoms (first - third trimester), the speed of their progress, the regularity and 
character of the menstrual cycle before pregnancy, and also the use of progestins, androgens and other medicaments during pregnancy.

During the physical examination we assess body mass index (BMI), blood pressure, we quantify the level of hirsutism, the presence of signs of virilization, cushingoid traits and we supplement this with a vaginal and rectal examination.

Laboratory examination is focussed on determining the level of testosterone, androstenedione, dehydroepiandrosterone and its sulphate, dihydrotestosterone, SHBG, cortisol and estrogens (estradiol, estriol).

Of the various imaging methods the method of choice is the ultrasound examination of the adrenal glands and ovaries that enables an assessment of the size of the formation, the distinguishing of their solid and cystic character, unilateral and bilateral affliction, or possible changes in the sex of the fetus as the case may be. During pregnancy, however, it might not be sufficiently clear, which is why a magnetic resonance can be used to precisely assess the local growth of the tumor, or the presence of metastasis.

Since the most frequent causes of hyperandrogenic states with pregnant women it pregnancy luteoma and hyperreactio luteinalis, the first step is to rule out all serious causes, particularly tumor causes that could threaten the patient's life.

\section{Conflict of Interest}

There is no conflict of interest.

\section{Acknowledgements}

This paper is dedicated to Prof. MUDr. RNDr. L. Stárka, DrSc., one of the leaders in the area of endocrinology. Work was supported by the grant No 303/08958 of Grant Agency of Czech Republic.

\begin{abstract}
Abreviations
SHBG, sex hormone binding globulin; DHEA, dehydroepiandrosterone; DHEAS, dehydroepiandrosterone sulfate; DHT, dihydrotestosterone; CYP 17,

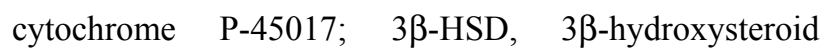
dehydrogenase; 17 $\beta$-HSD, 17 $\beta$-hydroxysteroid dehydrogenase; $\mathrm{CAH}$, congenital adrenal hyperandrogenemia; hCG, human chorionic gonadotropin; $\mathrm{E}$, estrone; $\mathrm{E}_{2}$, estradiol; $\mathrm{E}_{3}$, estriol; $\mathrm{FAD}$, placental aromatase deficiency; PCOS, polycystic ovary syndrome; US, ultrasound; CRH, corticotropin-releasing hormone; APGAR score, Dr. Virginia Apgar score; CT, computer tomography; BMI, body mass index; OHSS, ovarian hyperstimulation syndrome.
\end{abstract}

\section{References}

AL-RAMAHI M, LEADER A: Hyperreactio luteinalis associated with chronic renal failure. Hum Reprod 14: 416-418, 1999.

AYHAN A, TUNCER ZS, AKSU AT: Ovarian hyperstimulation syndrome associated with spontaneous pregnancy. Hum Reprod 11: 1600-1601, 1996.

AZMOUN L, DISALVO D: Pregnancy Luteoma. In: Endocrine Disorders in Pregnancy. SC YEN, RB JAFFE, RL BARBIERI (eds.), WB Saunders Company, Philadelphia, 1999, pp 785-811.

BARBIERI RL: Management of Endocrine Disorders in Pregnancy. SC YEN, RB JAFFE, RL BARBIERI (eds.), WB Saunders Company, Philadelphia, 1999.

BEN-CHETRIT A, GREENBLATT EM: Recurrent maternal virilization during pregnancy associated with polycystic ovarian syndrome: a case report and review of the literature. Hum Reprod 10: 3057-3060, 1995.

BETZOLD CM, HOOVER KL, SNYDER CL: Delayed lactogenesis II: a comparison of four cases. J Midwifery Womens Health 49: 132-137, 2004.

CHECK ML, BROWN T, CHECK JH: Recovery of spermatogenesis and successful conception after bone marrow transplant for acute leukaemia: case report. Hum Reprod 15: 83-85, 2000.

CHEN CH, CHEN IC, WANG YC, LIU JY, WU GJ, TZENG CR: Boy born after gender preselection following successive gestational androgen excess of maternal luteoma and female disorders of sex development. Fertil Steril 91: 2732 e5-7, 2009.

CHOI JR, LEVINE D, FINBERG H: Luteoma of pregnancy: sonographic findings in two cases. J Ultrasound Med 19: 877-881, 2000.

CLEMENT PB: Tumor-like lesions of the ovary associated with pregnancy. Int J Gynecol Pathol 12: 108-115, 1993. 
DAHL SK, THOMAS MA, WILLIAMS DB, ROBINS JC: Maternal virilization due to luteoma associated with delayed lactation. Fertil Steril 90: 2006 e17-9, 2008.

FOREST MG, ORGIAZZI J, TRANCHANT D, MORNEX R, BERTRAND J: Approach to the mechanism of androgen overproduction in a case of Krukenbery tumor responsible for virilization during pregnancy. $J$ Clin Endocrinol Metab 47: 428-434, 1978.

FOULK RA, MARTIN MC, JERKINS GL, LAROS RK: Hyperreactio luteinalis differentiated from severe ovarian hyperstimulation syndrome in a spontaneously conceived pregnancy. Am J Obstet Gynecol 176: 1300-1302; discussion 1302-1304, 1997.

HARADA N, OGAWA H, SHOZU M, YAMADA K: Genetic studies to characterize the origin of the mutation in placental aromatase deficiency. Am J Hum Genet 51: 666-672, 1992.

HOLT HB, MEDBAK S, KIRK D, GUIRGIS R, HUGHES I, CUMMINGS MH, MEEKING DR: Recurrent severe hyperandrogenism during pregnancy: a case report. J Clin Pathol 58: 439-442, 2005.

ILLINGWORTH PJ, JOHNSTONE FD, STEEL J, SETH J: Luteoma of pregnancy: masculinisation of a female fetus prevented by placental aromatisation. Br J Obstet Gynaecol 99: 1019-1020, 1992.

JOSHI R, DUNAIF A: Ovarian disorders of pregnancy. Endocrinol Metab Clin North Am 24: 153-169, 1995.

KAO HW, WU CJ, CHUNG KT, WANG SR, CHEN CY: MR imaging of pregnancy luteoma: a case report and correlation with the clinical features. Korean J Radiol 6: 44-46, 2005.

KOCHENOUR NK: Lactation suppression. Clin Obstet Gynecol 23: 1045-1059, 1980.

KURMAN RJ: Blaustein's pathology of the female genital tract. In: Blaustein's Pathology of the Female Genital Tract. Springer Verlag, New York, 1987.

LAMBERS DS, ROSENN B: Hyperreactio luteinalis complicating a normal singleton pregnancy. Am J Perinatol 13: 491-494, 1996.

MANGANIELLO PD, ADAMS LV, HARRIS RD, ORNVOLD K: Virilization during pregnancy with spontaneous resolution postpartum: a case report and review of the English literature. Obstet Gynecol Surv 50: 404-410, 1995.

MCCLAMROCK HD, ADASHI EY: Gestational hyperandrogenism. Fertil Steril 57: 257-274, 1992.

MILEWICH L, GOMEZ-SANCHEZ C, MADDEN JD, BRADFIELD DJ, PARKER PM, SMITH SL, CARR BR, EDMAN CD, MACDONALD PC: Dehydroisoandrosterone sulfate in peripheral blood of premenopausal, pregnant and postmenopausal women and men. J Steroid Biochem 9: 1159-1164, 1978.

NORWITZ ER: Endocrine diseases of pregnancy. In: Yen and Jaffe's Reproductive Endocrinology: Physiology, Pathophysiology and Clinical Management. J STRAUSS, RL BARBIERI (eds.), Elsevier Saunders, Philadelphia, 2004, pp 735-785.

PASCAL RR, GRECCO LA: Mucinous cystadenoma of the ovary with stromal luteinization and hilar cell hyperplasia during pregnancy. Hum Pathol 19: 179-180, 1988.

QUAGLIARELLO J, BLAUSTEIN A: Maternal virilization in pregnancy due to an unclassified sex-cord stromal neoplasm. Am J Obstet Gynecol 152: 570-572, 1985.

RIVAROLA MA, FOREST MG, MIGEON CJ: Testosterone, androstenedione and dehydroepiandrosterone in plasma during pregnancy and at delivery: concentration and protein binding. J Clin Endocrinol Metab 28: 34-40, 1968.

SCHENKER JG, EZRA Y: Complications of assisted reproductive techniques. Fertil Steril 61: 411-422, 1994.

SHORTLE BE, WARREN MP, TSIN D: Recurrent androgenicity in pregnancy: a case report and literature review. Obstet Gynecol 70: 462-466, 1987.

SPITZER RF, WHERRETT D, CHITAYAT D, COLGAN T, DODGE JE, SALLE JL, ALLEN L: Maternal luteoma of pregnancy presenting with virilization of the female infant. J Obstet Gynaecol Can 29: 835-840, 2007.

STERNBERG WH: Nonfunctioning ovarian neoplasms. In: The Ovary. HG GRADY (ed.), Williams and Wilkins, Baltimore, 1963, p. 209.

TAN ML, LAM SL, NADARAJAH S: Pregnancy luteoma presenting as ovarian torsion with rupture and intraabdominal bleeding. Singapore Med J 49: e78-81, 2008.

UEDA M, UEKI M: Ovarian tumors associated with pregnancy. Int J Gynaecol Obstet 55: 59-65, 1996. 
VULINK AJ, VERMES I, KUIJPER P, TEN CATE LN, SCHUTTER EM: Steroid cell tumour not otherwise specified during pregnancy: a case report and diagnostic work-up for virilization in a pregnant patient. Eur J Obstet Gynecol Reprod Biol 112: 221-227, 2004.

WAJDA KJ, LUCAS JG, MARSH WL JR: Hyperreactio luteinalis. Benign disorder masquerading as an ovarian neoplasm. Arch Pathol Lab Med 113: 921-925, 1989.

WANG YC, SU HY, LIU JY, CHANG FW, CHEN CH: Maternal and female fetal virilization caused by pregnancy luteomas. Fertil Steril 84: 509, 2005.

WY LA, CARLSON HE, KANE P, LI X, LEI ZM, RAO CV: Pregnancy-associated Cushing's syndrome secondary to a luteinizing hormone/human chorionic gonadotropin receptor-positive adrenal carcinoma. Gynecol Endocrinol 16: 413-417, 2002.

YOUNG RH, DUDLEY AG, SCULLY RE: Granulosa cell, Sertoli-Leydig cell, and unclassified sex cord-stromal tumors associated with pregnancy: a clinicopathological analysis of thirty-six cases. Gynecol Oncol 18: 181$205,1984$.

ZALEL Y, KATZ Z, CASPI B, BEN-HUR H, DGANI R, INSLER V: Spontaneous ovarian hyperstimulation syndrome concomitant with spontaneous pregnancy in a woman with polycystic ovary disease. Am J Obstet Gynecol 167: 122-124, 1992. 